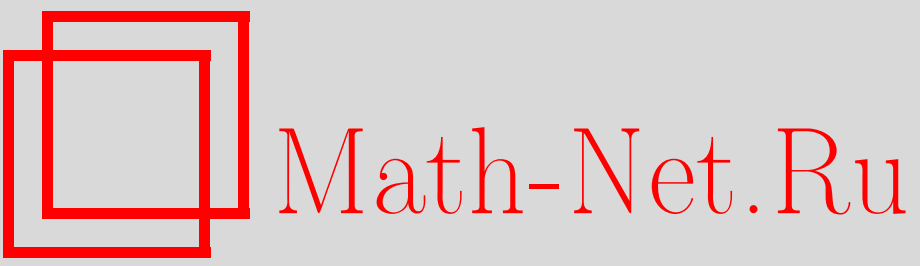

Ю. М. Лоскутов, Положительная определенность интенсивности гравитационного излучения в теории гравитации с ненулевой массой гравитона, ТMФ, 1996, том 107, номер 2, 329-343

DOI: https://doi.org/10.4213/tmf1159

Использование Общероссийского математического портала Math-Net.Ru подразумевает, что вы прочитали и согласны с пользовательским соглашением

http://www.mathnet.ru/rus/agreement

Параметры загрузки:

IP : 52.87 .193 .239

26 апреля 2023 г., 12:10:07

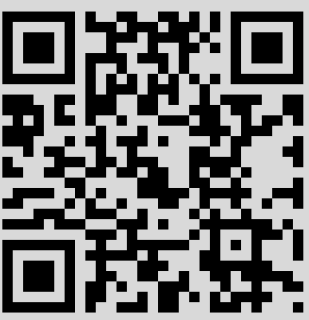


ТЕОРЕТИЧЕСКАЯ

И МАТЕМАТИЧЕСКАЯ

ФИЗИКА

Том 107, № 2

май, 1996

\author{
Ю.М. Лоскутов
}

\title{
ПОЛОЖИТЕЛЬНАЯ ОПРЕДЕЛЕННОСТЬ ИНТЕНСИВНОСТИ ГРАВИТАЦИОННОГО ИЗЛУЧЕНИЯ В ТЕОРИИ ГРАВИТАЦИИ С НЕНУЛЕВОЙ МАССОЙ ГРАВИТОНА
}

\begin{abstract}
В используемом варианте теории гравитации с ненулевой массой гравитона поток гравитационного излучения от произвольного пространственно-ограниченного источника является положительно-определенным. Установлена связь потерь энергии на излучение с работой источников. Показано, что полная работа содержит часть, обусловленную взаимодействием источника с полем излучения, и часть, обусловленную самодействием поля. В целом это и делает работу положительно-определенной. Получен общий вид спектрально-углового распределения излучения с учетом спиновых и поляризационных состояний. В случае сферически-симметричных источников вклад в излучение дают состояния с нулевым спином и с нулевой проекцией спина два на им-
\end{abstract} пульс.

\section{1. ВВЕДЕНИЕ}

Известно, сколь принципиальное значение имеет вопрос о наличии у таких частиц, как нейтрино, фотон, гравитон, массы покоя. Достаточно напомнить о роли массынейтрино в различных физических процессах и о роли массы фотона в электромагнитных явлениях. Теория в силу своего концептуального смысла сама по себе в принципе не может ответить на вопрос, обладает или не обладает массой покоя та или иная частица. В ней просто принимается то или иное соглашение. В зависимости от соглашения теория может дать лишь определенные следствия, подлежашие экспериментальной проверке. С другой стороны, экспериментально для фотонов и нейтрино удается установить (в рамках достигнутой точности) лишь верхнюю границу массы, т.е. снизу вопрос о ней всегда остается открытым. В случае электрослабых и электромагнитных взаимодействий введение в теорию массы (если природа наделила соответствуюшие частицы пусть очень малой, но конечной массой) не сопряжено с принципиальными трудностями и физически неприемлемыми следствиями. Иная ситуация имеет место в теории гравитации. Здесь введение в теорию массы гравитона, если таковой наделила его природа, наталкивается на определенные осложнения. Связаны они с тем, что включение в плотность лагранжиана $\mathcal{L}$ (и вытекаюшие уравнения) массового члена (будем называть его далее $\mu$-членом, подразумевая под $\mu$ массу покоя гравитона) требует, как это было показано, например, в [1], введения наряду с метрикой $g_{\alpha \beta}$ риманова пространства дополнительной метрики. По ряду соображений в качестве таковой выбирается (см. $[1,2,3])$ метрика $\gamma_{\alpha \beta}$ пространства Минковского. Это ведет к двум следствиям. 
Во-первых, утрачивается последовательность римановой геометрической интерпретации теории $[1,3]$, поскольку пространство с метрикой $\gamma_{\alpha \beta}$ приобретает смысл фундаментального пространства, в котором и реализуются гравитационные (как и все иные) процессы. Метрический тензор $g_{\alpha \beta}$ должен пониматься теперь как тензор, индуцируемый физическим гравитационным полем, определяемым в пространстве с метрикой $\gamma_{\alpha \beta}$. Риманову пространству придается тем самым смысл эффективного, индуцируемого физическим полем пространства. Это, однако, не лишает его практической значимости, поскольку и наблюдатель, и его приборы также подвержены действию поля, т.е. “погружены” в это эффективное пространство. Вместе с тем введение фундаментальной метрики $\gamma_{\alpha \beta}$ позволяет транспонировать мысленную операцию включения и выключения поля на все физические ситуации. Иначе говоря, введение $\gamma_{\alpha \beta}$ делает возможным выносить суждение о том, вызвано ли соответствуюшее явление действием гравитационного поля или же оно имеет место и в его отсутствие.

Во-вторых, наличие двух метрик делает процедуру включения $\mu$-члена в скалярную плотность $\mathcal{L}$ неоднозначной, если не сформулировать дополнительных требований, налагаемых на $\mathcal{L}$.

В [1] были рассмотрены различные варианты построения $\mathcal{L}$ с $\mu$-членом. Главный вывод, к которому пришли авторы [1], сводится к заключению о невозможности построения приемлемой, непротиворечивой теории гравитации с ненулевой массой гравитона. Однако, если бы этот вывод был справедлив, то мы имели бы уникальный случай в физике, когда путем только теоретических рассуждений доказывалось бы точное равенство нулю массы покоя гравитона. Такая теорема не может не вызвать сомнений. На чем же основан указанный вывод? Главным образом он базируется на том, что из-за наличия в составе поля наряду с компонентой, соответствующей спину два, скалярной компоненты, соответствуюшей нулевому спину, энергия свободного гравитационного поля приобретает отрицательный вклад. Это, в свою очередь, должно приводить, заключают авторы [1], к неустойчивостям источников поля.

Здесь надо уточнить, что неустойчивость вызывает не сама по себе отрицательная плотность энергии, а отрицательные вклады в поток энергии, уносимой им от источника на бесконечность. Например, в случае статического источника плотность энергии гравитационного поля (массивного или безмассового) вне источника всегда отрицательна, но она не приводит к неустойчивостям, т.к. от источника на бесконечность не уносится (поток его равен нулю). Если же в потоке появляются отрицательные вклады, то они, конечно, приведут к неустойчивости. Чтобы выяснить их природу и установить, не связано ли их происхождение с какими-то некорректностями, проведем соответствуюшие вычисления, пользуясь обшепринятыми методами. Забегая же вперед, скажем, что именно в этих методах и будет содержаться некорректность.

В обшем случае плотность лагранжиана поля с $\mu$-членом можно (см. [1]) представить в виде ${ }^{1)}$

$$
\mathcal{L}_{g} \equiv \mathcal{L}_{g}^{0}+\mu^{2} f(g, \gamma)
$$

где $\mathcal{L}_{g}^{0}$ - часть, не зависяшая от $\mu$. Так как согласно [1] вывод о наличии отрицательного вклада в плотность энергии (и, добавим, в плотность потока при обычно применяемых методах расчета) от выбора $f$ не зависит, возьмем $\mu$-член в форме, предложенной впер-

\footnotetext{
${ }^{1)}$ Всюду используется система единиц $c=\hbar=G=1$.
} 
вые в [3] (обоснование такой конструкции будет дано ниже в разделе 2),

$$
\mu^{2} f(g, \gamma) \equiv-\frac{\mu^{2}}{16 \pi}\left(\frac{1}{2} \tilde{g}^{\alpha \beta} \gamma_{\alpha \beta}-\sqrt{-g}-\sqrt{-\gamma}\right)
$$

где $\tilde{g}^{\alpha \beta} \equiv \sqrt{-g} g^{\alpha \beta}$. Плотность $\mathcal{L}_{g}^{0}$, учитьвая фундаментальность метрики $\gamma_{\alpha \beta}$, возьмем, обобшая [3], равной

$$
\mathcal{L}_{g}^{0} \equiv \frac{1}{16 \pi} \tilde{g}^{\varepsilon \lambda}\left(G_{\varepsilon \lambda}^{\alpha} G_{\alpha \beta}^{\beta}-G_{\varepsilon \beta}^{\alpha} G_{\lambda \alpha}^{\beta}\right),
$$

где тензор третьего ранга

$$
G_{\varepsilon \lambda}^{\alpha} \equiv \frac{1}{2} g^{\alpha \beta}\left(D_{\varepsilon} g_{\beta \lambda}+D_{\lambda} g_{\beta \varepsilon}-D_{\beta} g_{\varepsilon \lambda}\right)
$$

а $D_{\alpha}$ является ковариантной производной в пространстве Минковского ${ }^{2)}$; в галилеевых координатах $D_{\alpha}=\partial_{\alpha}$ и $G_{\varepsilon \lambda}^{\alpha}=\Gamma_{\varepsilon \lambda}^{\alpha}$. Плотность $\tau^{\varepsilon \lambda} \equiv-2\left(\delta \mathcal{L}_{g} / \delta g_{\varepsilon \lambda}\right)$ метрического тензора энергии-импульса массивного гравитационного поля в римановом пространстве будет в таком случае определяться выражением

$$
\tau^{\varepsilon \lambda} \equiv-\frac{1}{8 \pi \sqrt{-g}}\left[\sqrt{-g} \widetilde{R}^{\varepsilon \lambda}-\frac{1}{2} \tilde{g}^{\varepsilon \lambda} \widetilde{R}+\frac{1}{2} \mu^{2}\left(\sqrt{-g} \tilde{g}^{\varepsilon \lambda}+\tilde{g}^{\varepsilon \alpha} \tilde{g}^{\lambda \beta} \gamma_{\alpha \beta}-\frac{1}{2} \tilde{g}^{\varepsilon \lambda} \tilde{g}^{\alpha \beta} \gamma_{\alpha \beta}\right)\right]
$$

здесь $\widetilde{R}^{\varepsilon \lambda} \equiv \sqrt{-g} R^{\varepsilon \lambda}, \widetilde{R} \equiv \sqrt{-g} R, R \equiv R_{\alpha \beta} g^{\alpha \beta}$, а $R_{\alpha \beta}$ - тензор Риччи. Плотность же $t_{g}^{\varepsilon \lambda} \equiv-2\left(\delta \mathcal{L}_{g} / \delta \gamma_{\varepsilon \lambda}\right)$ метрического тензора энергии-импульса поля в пространстве Минковского будет равной

$$
t_{g}^{\varepsilon \lambda} \equiv 2 \sqrt{-\gamma}\left(\gamma^{\varepsilon \alpha} \gamma^{\lambda \beta}-\frac{1}{2} \gamma^{\varepsilon \lambda} \gamma^{\alpha \beta}\right) \frac{\delta \mathcal{L}_{g}}{\delta \tilde{g}^{\alpha \beta}}-\frac{1}{16 \pi}\left[J^{\varepsilon \lambda}-\mu^{2}\left(\tilde{g}^{\varepsilon \lambda}-\tilde{\gamma}^{\varepsilon \lambda}\right)\right],
$$

где

$$
J^{\varepsilon \lambda} \equiv D_{\alpha} D_{\beta}\left(\gamma^{\alpha \varepsilon} \tilde{g}^{\beta \lambda}+\gamma^{\alpha \lambda} \tilde{g}^{\beta \varepsilon}-\gamma^{\varepsilon \lambda} \tilde{g}^{\alpha \beta}-\gamma^{\alpha \beta} \tilde{g}^{\varepsilon \lambda}\right), \quad \tilde{\gamma}^{\varepsilon \lambda} \equiv \sqrt{-\gamma} \gamma^{\varepsilon \lambda} .
$$

Вне источника или в случае свободного поля плотность $\tau^{\varepsilon \lambda}$ обрашается в нуль, т.к. вариационная производная $\delta \mathcal{L}_{g} / \delta g_{\varepsilon}$ дает одновременно динамические уравнения поля $\left(\delta \mathcal{L}_{g} / \delta g_{\varepsilon \lambda}=0\right)$. При наличии источника уравнения имеют вид

$$
\tau^{\varepsilon \lambda}+T^{\varepsilon \lambda}=0
$$

где $T^{\varepsilon \lambda} \equiv-2\left(\delta \mathcal{L}_{m} / \delta g_{\varepsilon \lambda}\right)$ - плотность тензора энергии-импульса вешества ${ }^{3)}$. Заметим, кстати, что в отсутствие $\mu$-члена уравнение (7) переходит в обычное уравнение Гильберта-Эйнштейна для безмассового поля. Определим (подобно тому как это делалось в $[1,3])$ гравитационные потенциалы поля в пространстве Минковского отклонениями

$$
\widetilde{\Phi}^{\varepsilon \lambda} \equiv \sqrt{-\gamma} \Phi^{\varepsilon \lambda} \equiv \tilde{g}^{\varepsilon \lambda}-\tilde{\gamma}^{\varepsilon \lambda}
$$

\footnotetext{
${ }^{2)}$ Представленная здесь структура $\mathcal{L}_{g}^{0}$ является общековариантной. Авторы [3] не нашли, что возможна общековариантная формулировка теории с $\mu$-членом, допускающая любой выбор координат и соответственно метрики Минковского; поэтому в [3] построение теории велось на базе диагональной метрики $\gamma_{\alpha \beta}$ (в галилеевых координатах). Более того, отсутствие общей ковариантности теории подчеркивается ими как необходимая дань введению (в силу включения $\mu$-члена) метрики Минковского. Об общековариантном подходе к построению теории с $\mu$-членом см. ниже в разделе 2 .

3) Для удобства термином "вещество" условимся называть все формы материи, за исключением гравитационного поля.
} 
Тогда в силу динамических уравнений вещества

$$
\nabla_{\lambda} T^{\varepsilon \lambda}=0
$$

где $\nabla_{\lambda}$ - ковариантная производная в римановом пространстве, из (7) будет следовать полевое условие

$$
D_{\lambda} \tilde{g}^{\varepsilon \lambda}=D_{\lambda} \widetilde{\Phi}^{\varepsilon \lambda}=0
$$

Если учесть, что на уравнениях поля $\delta \mathcal{L}_{g} / \delta \tilde{g}^{\alpha \beta}=0$, т.е. вне источника, тождество (6) становится адекватным $(7)-$ без $T^{\varepsilon \lambda}$ - уравнением

$$
J^{\varepsilon \lambda}+\mu^{2} \widetilde{\Phi}^{\varepsilon \lambda}=16 \pi t_{g}^{\varepsilon \lambda}
$$

и принять во внимание равенство $D_{\lambda} t_{g}^{\varepsilon \lambda}=0$, то (10) легко получить и отсюда, т.к. $D_{\lambda} J^{\varepsilon \lambda} \equiv 0$. Пользуясь $[4,5]$, можно убедиться, что полевое условие (10) исключает из состава гравитационного поля состояния, соответствуюшие спинам 1 и $0^{\prime}$, преобразуя его в скалярно-тензорную смесь со спиновыми состояниями 2 и 0.

Прибегая теперь к общепринятой (см., например, [1]) процедуре, разложим (5) в ряд по степеням поля $\Phi^{\varepsilon \lambda}$ с точностью до квадратичных членов включительно. Тогда получим

$$
\begin{aligned}
\tau^{\varepsilon \lambda} \simeq & \frac{\sqrt{-\gamma}}{16 \pi}\left\{-\gamma^{\alpha \beta} D_{\alpha} D_{\beta} \Phi^{\varepsilon \lambda}-\mu^{2} \Phi^{\varepsilon \lambda}+\right. \\
& +\frac{1}{2}\left(\gamma^{\varepsilon \alpha} \gamma^{\lambda \beta}-\frac{1}{2} \gamma^{\varepsilon \lambda} \gamma^{\alpha \beta}\right)\left(D_{\alpha} \Phi_{\tau}^{\nu} D_{\beta} \Phi_{\nu}^{\tau}-\frac{1}{2} D_{\alpha} \Phi D_{\beta} \Phi\right)- \\
& -\mu^{2}\left[\Phi_{\alpha}^{\varepsilon} \Phi^{\lambda \alpha}-\frac{1}{4} \gamma^{\varepsilon \lambda}\left(\Phi_{\beta}^{\alpha} \Phi_{\alpha}^{\beta}-\frac{1}{2} \Phi \Phi\right)\right]- \\
& -\gamma^{\varepsilon \beta} D_{\alpha} \Phi^{\lambda \nu} D_{\beta} \Phi_{\nu}^{\alpha}-\gamma^{\lambda \alpha} D_{\alpha} \Phi^{\beta \nu} D_{\beta} \Phi_{\nu}^{\varepsilon}+\gamma^{\alpha \beta} D_{\alpha} \Phi_{\nu}^{\varepsilon} D_{\beta} \Phi^{\lambda \nu}+ \\
& \left.+\frac{1}{2} \gamma^{\varepsilon \lambda} D_{\alpha} \Phi_{\nu}^{\beta} D_{\beta} \Phi^{\alpha \nu}+D_{\alpha} \Phi^{\varepsilon \beta} D_{\beta} \Phi^{\lambda \alpha}-\Phi^{\alpha \beta} D_{\alpha} D_{\beta} \Phi^{\varepsilon \lambda}\right\},
\end{aligned}
$$

где $\Phi \equiv \Phi_{\alpha}^{\alpha} \equiv \Phi^{\alpha \beta} \gamma_{\alpha \beta}$, а индексы у $\Phi^{\varepsilon \lambda}$ опускаются и поднимаются с помошью метрического тензора $\gamma_{\alpha \beta}$. Если в том же приближении записать плотность тензора $t_{g}^{\varepsilon \lambda}$ в пространстве Минковского, то она окажется тождественной квадратичной форме в (12). Это дает основание считать, что в соответствуюшем приближении плотность тензора энергии-импульса массивного гравитационного поля в целом определяется в пространстве Минковского квадратичной формой в $(12)$, которую обозначим через $t_{2}^{\varepsilon \lambda}$; таким образом,

$$
\tau^{\varepsilon \lambda} \equiv \frac{\sqrt{-\gamma}}{16 \pi}\left(-\gamma^{\alpha \beta} D_{\alpha} D_{\beta} \Phi^{\varepsilon \lambda}-\mu^{2} \Phi^{\varepsilon \lambda}\right)+t_{2}^{\varepsilon \lambda}
$$

Теперь легко видеть, что уравнение (11), если в нем учесть (10), адекватно уравнению $\tau^{\varepsilon \lambda}=0$, т.е. дальнейший анализ можно строить на любом из них.

Перейдем, пользуясь этим, к рассмотрению обычно применяемого метода получения плотности тензора энергии-импульса $t_{\mathrm{rad}}^{\varepsilon \lambda}$ nоля излучения источником. Первым и основным шагом на пути его построения является представление потенциалов $\Phi^{\varepsilon \lambda}$ в виде 
суммы $\Phi^{\varepsilon \lambda}=\stackrel{(0)}{\Phi} \Phi^{\varepsilon \lambda}+\stackrel{(1)}{\Phi} \Phi^{\varepsilon \lambda}$, в которой основной член подчиняется уравнению $(7)$ нулевого приближения

$$
\square \stackrel{(0)}{\Phi}{ }^{\varepsilon \lambda}+\mu^{2} \stackrel{(0)}{\Phi}^{\varepsilon \lambda}=16 \pi \stackrel{(0)}{T}^{\varepsilon \lambda}
$$

а поправочный $(\stackrel{(1)}{\Phi} \varepsilon \lambda)$ - уравнению следуюшего порядка, в правую часть которого войдет и квадратичная по полю форма с заменой в ней $\Phi^{\varepsilon \lambda}$ на $\stackrel{(0)}{\Phi} \Phi^{\varepsilon \lambda}$, т.е. $\stackrel{(0)}{t} \underset{2}{\varepsilon \lambda}$. Таким образом, вне источника связанная с полем излучения линейная часть $\tau^{\varepsilon \lambda}$ обратится в нуль и плотность $t_{\text {rad }}^{\varepsilon \lambda}$ идентифищируется в плотностью $t_{2}^{\varepsilon \lambda}$, построенной на решениях уравнения нулевого приближения.

Будем считать, что источник квазистационарен. Тогда вместо $t_{\text {rad }}^{\varepsilon \lambda}$ моно рассматривать усредненное по времени его значение $\bar{t}_{\text {rad }}^{\varepsilon \lambda}$ (практически так всегда и поступали). В таком случае члены $t_{\mathrm{rad}}^{\varepsilon \lambda}$, сводимые (при взятой точности) с помощњю полевого условия (10) или иным способом к форме 4-дивергенции (например, $D_{\alpha} \stackrel{(0)}{\Phi} \varepsilon^{\varepsilon} D_{\beta} \stackrel{(0)}{\Phi} \lambda \alpha=$ $D_{\alpha}\left(\stackrel{(0)}{\Phi} \varepsilon^{\varepsilon \beta} D_{\beta} \stackrel{(0)}{\Phi} \lambda \alpha\right)$ и др.), не будут давать вклада в $\bar{t}_{\mathrm{rad}}^{\varepsilon \lambda}$. Поэтому они в $t_{\mathrm{rad}}^{\varepsilon \lambda}$ могут быть опушены. Кроме того, пользуясь (14) вне источника, можно устранить из $t_{\mathrm{rad}}^{\varepsilon \lambda}$ и ряд других членов, например

$$
\begin{gathered}
\gamma^{\alpha \beta} D_{\alpha} \stackrel{(0)}{\Phi} \underset{\nu}{\varepsilon} D_{\beta} \stackrel{(0)}{\Phi}^{\lambda \nu}-\mu^{2} \stackrel{(0)}{\Phi}_{\nu}^{\varepsilon} \stackrel{(0)}{\Phi} \lambda \nu=D_{\alpha}\left(\gamma^{\alpha \beta} \stackrel{(0)}{\Phi} \underset{\nu}{\varepsilon} D_{\beta} \stackrel{(0)}{\Phi} \lambda \nu\right)- \\
-\stackrel{(0)}{\Phi} \underset{\nu}{\varepsilon}\left(\gamma^{\alpha \beta} D_{\alpha} D_{\beta} \stackrel{(0)}{\Phi}^{\lambda \nu}+\mu^{2} \stackrel{(0)}{\Phi}^{\lambda \nu}\right) .
\end{gathered}
$$

В итоге получим

$$
t_{\mathrm{rad}}^{\varepsilon \lambda}=\frac{\sqrt{-\gamma}}{32 \pi} \gamma^{\varepsilon \alpha} \gamma^{\lambda \beta}\left(D_{\alpha} \stackrel{(0)}{\Phi} \underset{\tau}{\nu} D_{\beta} \stackrel{(0)}{\Phi} \underset{\nu}{\tau}-\frac{1}{2} D_{\alpha} \stackrel{(0)}{\Phi} D_{\beta} \stackrel{(0)}{\Phi}\right)
$$

Таким образом, обычно применяемые методы приводят к выражению для потока излучения (в галилеевых координатах)

$$
\stackrel{(0)}{I}=-\frac{1}{32 \pi} \oint_{S \rightarrow \infty}\left[\partial_{0} \stackrel{(0)}{\Phi} \underset{\beta}{\alpha} \partial_{k} \stackrel{(0)}{\Phi} \underset{\alpha}{\beta}-\frac{1}{2} \partial_{0} \stackrel{(0)}{\Phi} \partial_{k} \stackrel{(0)}{\Phi}\right] d \sigma^{k}
$$

идентичному по форме потоку излучения гравитационного поля с нулевой массой ${ }^{4}$.

$$
(0)
$$

Представим решения $\stackrel{(0)}{\Phi} \varepsilon \lambda$ вдали от источника рядом Фурье

$$
\stackrel{(0)}{\Phi} \varepsilon \lambda \simeq \frac{1}{r} \sum_{\omega} a_{\omega}^{\varepsilon \lambda} \exp (-i \omega t+i \varkappa \mathbf{r}) \equiv \sum_{\omega} \stackrel{(0)}{\Phi} \underset{\omega}{\varepsilon \lambda},
$$

\footnotetext{
4) $\mathrm{K}$ виду (15) и соответственно (16) сводится, например, усредненное по времени выражение для псевдотензора поля излучения, приведенное в [6].
} 
где $\varkappa \equiv \mathbf{n} \eta \omega, \eta \equiv\left[1-(\mu / \omega)^{2}\right]^{1 / 2}, \mathbf{n} \equiv \mathbf{r} / r, \omega \equiv \nu \omega_{0}$, а $\omega_{0} \geq \mu$. Тогда можно перейти к спектрально-угловому распределению излучения

$$
\frac{d \stackrel{(0)}{I}}{d \Omega}=\frac{1}{16 \pi} \sum_{\nu=1}^{\infty} \omega^{2} \eta\left\{{ }_{a}^{*}{ }_{\lambda}^{\varepsilon}(\varkappa) a_{\varepsilon}^{\lambda}(\varkappa)-\frac{1}{2} \stackrel{*}{a}(\varkappa) a(\varkappa)\right\} .
$$

Учитьвая (при взятой точности) вытекающие из (10) равенства

$$
a_{0}^{0}=-\eta^{2} a_{3}^{3}, \quad a_{k}^{0}=-a_{0}^{k}=\eta a_{k}^{3},
$$

где индексу 3 сопоставлено направление импульса $\varkappa$, получим

$$
\begin{aligned}
\frac{d \stackrel{(0)}{I I}}{d \Omega}= & \frac{1}{8 \pi} \sum_{\nu=1}^{\infty} \omega^{2} \eta\left\{\left|a_{2}^{1}\right|^{2}+\frac{1}{4}\left|a_{1}^{1}-a_{2}^{2}\right|^{2}+\frac{\mu^{2}}{\omega^{2}}\left(\left|a_{3}^{1}\right|^{2}+\left|a_{3}^{2}\right|^{2}\right)+\right. \\
& \left.+\frac{\mu^{4}}{4 \omega^{4}}\left|a_{3}^{3}\right|^{2}-\frac{\mu^{2}}{4 \omega^{2}}\left[\left(a_{1}^{*}+a_{2}^{*}\right) a_{3}^{3}+\left(a_{1}^{1}+a_{2}^{2}\right)_{3}^{*}\right]\right\} .
\end{aligned}
$$

Отсюда видно, что отрицательньй вклад в поток излучения, полученного общепринятыми методами, действительно возникает и обязан своим появлением ненулевой массе гравитона (при $\mu=0$ спектрально-угловое распределение преобразуется в известное (см., например, [6]) и становится положительно-определенным). В частности, для сферически-симметричного источника, когда отличными от нуля остаются $a_{1}^{1}=a_{2}^{2}=a_{3}^{3}$, имеем

$$
\frac{d \stackrel{(0)}{I}}{d \Omega}=-\frac{\mu^{2}}{8 \pi} \sum_{\nu=1}^{\infty} \eta\left(1-\frac{\mu^{2}}{4 \omega^{2}}\right)\left|a_{3}^{3}\right|^{2}
$$

$(0)$

Чтобы выяснить, какой вклад в $d I / d \Omega$ дают отдельные спиновые состояния поля, воспользуемся результатами $[4,5]$ и разложим $a^{\varepsilon \lambda}$ по неприводимым представлениям, соответствуюшим спинам 2 и 0 . Учитывая значения проекционных операторов в импульсном пространстве

$$
\begin{aligned}
& \left(P_{2}\right)_{\alpha \beta}^{\varepsilon \lambda} \equiv \frac{3}{2}\left(Q_{\alpha}^{\varepsilon} Q_{\beta}^{\lambda}+Q_{\beta}^{\varepsilon} Q_{\alpha}^{\lambda}\right)-Q_{\alpha \beta} Q^{\varepsilon \lambda}, \\
& \left(P_{0}\right)_{\alpha \beta}^{\varepsilon \lambda} \equiv Q_{\alpha \beta} Q^{\varepsilon \lambda}, \quad Q^{\varepsilon \lambda} \equiv \frac{1}{\sqrt{3}}\left(\gamma^{\varepsilon \lambda}-\frac{\varkappa^{\varepsilon} \varkappa^{\lambda}}{\varkappa^{2}}\right)
\end{aligned}
$$

получим

$$
c^{\varepsilon \lambda} \equiv \frac{1}{3}\left(\gamma^{\varepsilon \lambda}-\frac{\varkappa^{\varepsilon} \varkappa^{\lambda}}{\varkappa^{2}}\right) a, \quad c=a, \quad b^{\varepsilon \lambda} \equiv a^{\varepsilon \lambda}-c^{\varepsilon \lambda}
$$

где $b^{\varepsilon \lambda}$ относятся к состояниям со спином 2 , а $c^{\varepsilon \lambda}$ - к состоянию с нулевым спином. Независимые амплитуды $b_{2}^{1}=a_{2}^{1},\left(b_{1}^{1}-b_{2}^{2}\right)=\left(a_{1}^{1}-a_{2}^{2}\right), b_{3}^{1}=a_{3}^{1}, b_{3}^{2}=a_{3}^{2}$ и $b_{3}^{3}=a_{3}^{3}-\left(\omega^{2} / 3 \varkappa^{2}\right) a$ будут отвечать соответственно двум поперечно-поперечным (связанным с проекциями $s_{3}= \pm 2$ спина 2$)$, двум поперечно-продольным (связанным с проекциями $s_{3}= \pm 1$ ) и 
одному продольно-продольному (связанному с проекцией $s_{3}=0$ ) состояниям, а амплитуда $c=a$ будет связана со скалярной примесью $(s=0)$. Таким образом, вместо $(20)$ получим

$$
\frac{d \stackrel{(0)}{I}}{d \Omega}=\frac{1}{8 \pi} \sum_{\nu=1}^{\infty} \omega^{2} \eta\left\{\left|b_{2}^{1}\right|^{2}+\frac{1}{4}\left|b_{1}^{1}-b_{2}^{2}\right|^{2}+\frac{\mu^{2}}{\omega^{2}}\left(\left|b_{3}^{1}\right|^{2}+\left|b_{3}^{2}\right|^{2}\right)+\frac{3 \mu^{4}}{4 \omega^{4}}\left|b_{3}^{3}\right|^{2}-\frac{1}{12}|c|^{2}\right\}
$$

Отсюда видно, что отрицательный вклад в поток излучения дает (как и отмечалось в [1]) только скалярная примесь.

Теперь, имея полную картину обычно принятых вычислений, обратим внимание на допущенную некорректность использованного метода расчетов и введения понятия плотности тензора энергии-импульса гравитационного поля излучения. Очевидно, что если она могла возникнуть, то только в самом начале, при выводе (16), т.к. все дальнейшее является лишь раскрытием результата (16). Сам результат (16), в свою очередь, является следствием применения метода последовательных приближений. В нем-то и содержится некорректность. Действительно, подчинение потенциалов $\Phi^{\varepsilon \lambda}$ в нулевом приближении линейному уравнению (14) с физической точки зрения означает, что пространство, в котором распространяются сопоставляемые нестатическому полю гравитоны, идентифицируется с пространством Минковского. Это отчетливо видно из того, что каждая фурье-компонента поля вне источника должна в силу (16) подчиняться линейному уравнению

$$
\square \stackrel{(0)}{\Phi} \underset{\omega}{\varepsilon \lambda}+\mu^{2} \stackrel{(0)}{\Phi} \underset{\omega}{\varepsilon \lambda}=0,
$$

приводящему к связи $\gamma_{\alpha \beta} \varkappa^{\alpha} \varkappa^{\beta}=\mu^{2}$. Однако согласиться с этим невозможно, т.к. реально гравитоны распространяются в римановом пространстве, индуцируемом как полями $\stackrel{(1)}{\Phi} \varepsilon \lambda$, так и собственным полем, т.е. импульсы $\varkappa^{\alpha}$ реально должны подчиняться связи $g_{\alpha \beta} \varkappa^{\alpha} \varkappa^{\beta}=\mu^{2}$. Примененный метод вычислений игнорирует это обстоятельство и потому приводит к некорректным следствиям. В разделе 3 будет развит иной подход, учитывающий факт распространения гравитонов в пространстве с римановой метрикой. Как окажется, это устранит отрицательньй вклад в поток и сделает его положительно-определенным. Тем самым снимутся возражения и утверждения [1] о невозможности построения непротиворечивой теории гравитации с ненулевой массой гравитона. Ниже излагаются ее основные моменты.

\section{2. ОСНОВНЫЕ УРАВНЕНИЯ ТЕОРИИ ГРАВИТАЦИИ С НЕНУЛЕВОЙ МАССОЙ ГРАВИТОНА}

Как уже отмечалось выше, включение в теорию гравитации массы гравитона, если таковой наделила его природа, влечет за собой (см. $[1,3])$ необходимость включения в нее наряду с римановой еше одной метрики. По ряду соображений (высокая степень согласия экспериментальных данных и теоретических предсказаний электродинамики, базируюшейся на понятиях пространства Минковского; многочисленные наблюдения, свидетельствуюшие об евклидовости трехмерного пространства и т.д.) наиболее целесообразным представляется идентифицировать эту метрику с метрикой пространства Минковского. В таком случае она приобретает фундаментальный смысл, а метрика $g_{\alpha \beta}$ 
оказывается вторичной, индуцируемой физическим гравитационным полем. Постулирование метрики Минковского в качестве фундаментальной гарантирует сушествование независимых законов сохранения энергии, импульса и момента количества движения замкнутых систем, т.к. пространство Минковского допускает 10-параметрическую группу Пуанкаре.

Хотя теперь динамические процессы естественнее трактовать как процессы, протекающие в пространстве Минковского под воздействием гравитационного поля, тем не менее движение вещества (см. сноску 3) под действием поля можно отождествить с его свободным движением в индуцируемом этим полем римановом пространстве. Это значит, что если плотность $\mathcal{L}_{M}$ лагранжиана вещества в пространстве Минковского (в пренебрежении гравитационными полями) содержала метрику $\gamma_{\alpha \beta}$, то при учете гравитационного поля в $\mathcal{L}_{M}$ вместо $\gamma_{\alpha \beta}$ войдут метрические коэффициенты $g_{\alpha \beta}$ риманова пространства, т.е. [7-10]

$$
\mathcal{L}_{M}\left(\tilde{\gamma}^{\alpha \beta}(x), A_{(\beta)}^{(\alpha)}(x), D_{\lambda} A_{(\beta)}^{(\alpha)}(x)\right) \rightarrow \mathcal{L}_{M}\left(\tilde{g}^{\alpha \beta}(x), A_{(\beta)}^{(\alpha)}(x), \nabla_{\lambda} A_{(\beta)}^{(\alpha)}(x)\right)
$$

Здесь $A_{(\beta)}^{(\alpha)}$ - тензорные (типа $\left.\left(\begin{array}{l}p \\ q\end{array}\right)\right)$ поля вешества с $(\alpha) \equiv \alpha_{1}, \alpha_{2}, \ldots, \alpha_{p}, \quad(\beta) \equiv \beta_{1}$, $\beta_{2}, \ldots, \beta_{q}$, а плотность $\widetilde{\Phi}^{\alpha \beta}(x)$ гравитационного поля в пространстве Минковского идентифицируется (по аналогии с [1-3]) с разностью $\tilde{g}^{\alpha \beta}-\tilde{\gamma}^{\alpha \beta}-$ см. (8). Как видно, $\mathcal{L}_{M}$ оказывается полностью геометризованной (в пространстве с метрикой $g_{\alpha \beta}$ ) плотностью, ибо не содержит метрики $\gamma_{\alpha \beta}$ явно. В [7-10] сопоставление (24) названо принципом геометризации.

Введем, как это сделано в [7-10], группу инфинитезимальных калибровочных преобразований полей

$$
\begin{aligned}
\delta_{\varepsilon} \tilde{g}^{\alpha \beta} & \equiv \delta_{\varepsilon} \widetilde{\Phi}^{\alpha \beta} \equiv \tilde{g}^{\alpha \lambda} D_{\lambda} \varepsilon^{\beta}+\tilde{g}^{\beta \lambda} D_{\lambda} \varepsilon^{\alpha}-D_{\lambda}\left(\varepsilon^{\lambda} \tilde{g}^{\alpha \beta}\right) \\
\delta_{\varepsilon} A_{(\beta)}^{(\alpha)} & \equiv F_{(\beta)(\tau) \mu}^{(\alpha) \nu} A_{(\sigma)}^{(\tau)} D_{\nu} \varepsilon^{\mu}-\varepsilon^{\lambda} D_{\lambda} A_{(\beta)}^{(\alpha)}
\end{aligned}
$$

где $\varepsilon^{\alpha}(x)$ является групповым параметром, структурные константы

$$
\begin{aligned}
F_{(\beta)(\tau) \mu}^{(\alpha)(\sigma) \nu} \equiv & \sum_{m=1}^{p} \delta_{\beta_{1}}^{\sigma_{1}} \cdots \delta_{\beta_{q}}^{\sigma_{q}} \delta_{\tau_{1}}^{\alpha_{1}} \cdots \delta_{\tau_{m}}^{\alpha_{m}=\nu} \cdots \delta_{\tau_{p}}^{\alpha_{p}} \delta_{\mu}^{\alpha_{m}}- \\
& -\sum_{m=1}^{q} \delta_{\tau_{1}}^{\alpha_{1}} \cdots \delta_{\tau_{p}}^{\alpha_{p}} \delta_{\beta_{1}}^{\sigma_{1}} \cdots \delta_{\beta_{m}=\mu}^{\sigma_{m}} \cdots \delta_{\beta_{q}}^{\sigma_{q}} \delta_{\beta_{m}}^{\nu}
\end{aligned}
$$

а операторы $\delta_{\varepsilon}$ образуют алгебру Ли и удовлетворяют тождествам Якоби. Тогда можно убедиться, что на этой группе скалярная плотность $\mathcal{L}_{M}$ изменяется не иначе чем на дивергентный член.

Естественно потребовать, чтобы и плотность лагранжиана гравитационного поля (без массового члена) также изменялась на этой группе не иначе чем на дивергенцию. Это требование, сформулированное впервые в [7-10], названо калибровочным принципом. Единственной (доказательство единственности см. в [7]) скалярной относительно общекоординатных преобразований плотностью $\mathcal{L}_{g}^{0}$, удовлетворяющей принципу минимальности и калибровочному принципу, оказывается структура (3). Таким образом, предложенная в [3] форма $\mathcal{L}_{g}^{0}$ получает не только ковариантное обобшение, но и находит 
обоснование как единственно возможная структура. И то, и другие имеет принципиальное значение для теории. Если физическое гравитационное поле идентифицировать со смесью $^{5)}$, отвечаюшей спиновым состояниям 2 и 0 , то единственно возможной структурой подключаемого к $\mathcal{L}_{g}^{0}$ массового члена (также удовлетворяюшей свойству скалярной относительно обшекоординатных преобразований плотности) будет структура (2), ибо только благодаря ей возникает полевое условие (10), исключающее из состава поля состояния со спинами 1 и 0'. Она же, кстати, гарантирует тождественное обрашение в нуль полной плотности $\mathcal{L}_{g}$ в отсутствие поля, что физически необходимо. Стало быть, и форма (2), предложенная впервые в [3], находит обоснование.

Включение в $\mathcal{L}_{g}$ массового члена нарушает группу калибровочных преобразований: при выполнении (10) массовый член будет изменяться на дивергенцию лишш на подмножестве параметров $\varepsilon^{\lambda}(x)$, удовлетворяющих уравнению

$$
g^{\alpha \beta} D_{\alpha} D_{\beta} \varepsilon^{\lambda}=0 .
$$

Аналогичное нарушение группы калибровочных преобразований имеет место и в электродинамике с ненулевой массой фотона.

Суммируя все сказанное, получаем однозначно определенное выражение для плотности лагранжиана вещества и гравитационного поля [7-10]

$$
\mathcal{L} \equiv \mathcal{L}_{M}+\frac{1}{16 \pi} \tilde{g}^{\varepsilon \lambda}\left(G_{\varepsilon \lambda}^{\alpha} G_{\alpha \beta}^{\beta}-G_{\varepsilon \beta}^{\alpha} G_{\lambda \alpha}^{\beta}\right)-\frac{\mu^{2}}{16 \pi}\left(\frac{1}{2} \tilde{g}^{\alpha \beta} \gamma_{\alpha \beta}-\sqrt{-g}-\sqrt{-\gamma}\right) .
$$

Система уравнений вешества и гравитационного поля примет тогда вид

$$
\frac{\delta \mathcal{L}}{\delta A_{(\lambda)}^{(\varepsilon)}} \equiv \frac{\delta \mathcal{L}_{M}}{\delta A_{(\lambda)}^{(\varepsilon)}}=0, \quad \frac{\delta \mathcal{L}}{\delta \tilde{g}^{\varepsilon \lambda}}=0 .
$$

На уравнениях вешества должно также иметь место уравнение (9). Если число независимых уравнений вешества в (26) окажется равным четырем, то их полностью можно заменить уравнениями (9) или условиями (10), т.к. (9) и (10) взаимозаменяемы. Такая ситуация реализуется для вешества, характеристиками которого могут служить скорости $u^{k}$ его элементов, плотность $\rho$ и давление $p$. Только в таких случаях система уравнений (26) трансформируется к виду (см. также [3, 7-10])

$$
\begin{gathered}
R^{\varepsilon \lambda}-\frac{1}{2} g^{\varepsilon \lambda} R+\frac{1}{2} \mu^{2}\left[g^{\varepsilon \lambda}+\left(g^{\varepsilon \alpha} g^{\lambda \beta}-\frac{1}{2} g^{\varepsilon \lambda} g^{\alpha \beta}\right) \gamma_{\alpha \beta}\right]=\frac{8 \pi}{\sqrt{-g}} T^{\varepsilon \lambda}, \\
D_{\lambda} \tilde{g}^{\varepsilon \lambda}=0 .
\end{gathered}
$$

Дополнив (27) уравнением состояния вешества $p=p(\rho)$, получим полную систему уравнений материи для определения всех пятнадцати его характеристик $\left(u^{k}, \rho, p\right.$ и $\left.\tilde{g}^{\varepsilon \lambda}\right)$.

Надо заметить, что при $\mu \rightarrow 0$ динамические (верхние из (27)) уравнения поля становятся вырожденными по метрике $\gamma_{\alpha \beta}$ пространства Минковского. Однако хорошо известно, что снятие вырождения может приводить к качественно новым физическим эффектам, скрытым в уравнениях с вырождением. Поэтому, даже если бы масса гравитона

\footnotetext{
5) Включение в состав поля состояний со спинами 1 и $0^{\prime}$ или одного из них вело бы к физически неприемлемым следствиям, вроде того как отсутствие в решениях уравнений для потенциалов поля предельного перехода при $\mu \rightarrow 0$ (см., например, [1]).
} 
в точности равнялась нулю, уравнения следовало бы дополнить членом, снимаюшим их вырождение по $\gamma_{\alpha \beta}$, и лиш после этого проводить расчеты, устремляя $\mu$ к нулю только на конечном этапе (см. [11]). В [12], в частности, показано, что такое снятие вырождения разносит особенности метрических коэффициентов $g_{00}^{-1}$ и $g_{11}$ (при $d t^{2}$ и $d r^{2}$ ) в статической сферически-симметричной задаче, приводя к невозможности проникновения падающих частиц под сферу Шваршшильда.

Убедимся теперь, что полученная система уравнений не ведет к противоречиям, т.е. плотность потока излучения массивных гравитонов является положительно-определенной.

\section{3. ПЛОТНОСТЬ ПОТОКА ИЗЛУЧЕНИЯ МАССИВНЫХ ГРАВИТОНОВ И ЕГО ПОЛОЖИТЕЛЬНАЯ ОПРЕДЕЛЕННОСТЬ}

Тождественными преобразованиями динамические уравнения (27) можно привести к виду

$$
\tilde{\gamma}^{\alpha \beta} D_{\alpha} D_{\beta} \widetilde{\Phi}^{\varepsilon \lambda}+\mu^{2} \sqrt{-\gamma} \widetilde{\Phi}^{\varepsilon \lambda}=16 \pi \sqrt{-g}\left(T^{\varepsilon \lambda}+t^{\varepsilon \lambda}\right),
$$

где

$$
\begin{aligned}
16 \pi \sqrt{-g} t^{\varepsilon \lambda} \equiv & \frac{1}{2}\left(\tilde{g}^{\varepsilon \alpha} \tilde{g}^{\lambda \beta}-\frac{1}{2} \tilde{g}^{\varepsilon \lambda} \tilde{g}^{\alpha \beta}\right)\left(\tilde{g}_{\nu \sigma} \tilde{g}_{\tau \varkappa}-\frac{1}{2} \tilde{g}_{\tau \sigma} \tilde{g}_{\nu \varkappa}\right) D_{\alpha} \widetilde{\Phi}^{\tau \sigma} D_{\beta} \widetilde{\Phi}^{\nu \varkappa}- \\
& -\mu^{2}\left[\sqrt{-g} \tilde{g}^{\varepsilon \lambda}-\sqrt{-\gamma} \widetilde{\Phi}^{\varepsilon \lambda}+\left(\tilde{g}^{\varepsilon \alpha} \tilde{g}^{\lambda \beta}-\frac{1}{2} \tilde{g}^{\varepsilon \lambda} \tilde{g}^{\alpha \beta}\right) \gamma_{\alpha \beta}\right]- \\
& -\tilde{g}^{\varepsilon \beta} \tilde{g}_{\tau \sigma} D_{\alpha} \widetilde{\Phi}^{\lambda \sigma} D_{\beta} \widetilde{\Phi}^{\alpha \tau}-\tilde{g}^{\lambda \alpha} \tilde{g}_{\tau \sigma} D_{\alpha} \widetilde{\Phi}^{\beta \sigma} D_{\beta} \widetilde{\Phi}^{\varepsilon \tau}+ \\
& +\tilde{g}^{\alpha \beta} \tilde{g}_{\tau \sigma} D_{\alpha} \widetilde{\Phi}^{\varepsilon \tau} D_{\beta} \widetilde{\Phi}^{\lambda \sigma}+\frac{1}{2} \tilde{g}^{\varepsilon \lambda} \tilde{g}_{\tau \sigma} D_{\alpha} \widetilde{\Phi}^{\sigma \beta} D_{\beta} \widetilde{\Phi}^{\alpha \tau}+ \\
& +D_{\alpha} \widetilde{\Phi}^{\varepsilon \beta} D_{\beta} \widetilde{\Phi}^{\lambda \alpha}-\widetilde{\Phi}^{\alpha \beta} D_{\alpha} D_{\beta} \widetilde{\Phi}^{\varepsilon \lambda}
\end{aligned}
$$

а $\tilde{g}_{\varepsilon \lambda} \equiv g_{\varepsilon \lambda} / \sqrt{-g}$. Если источник $T^{\varepsilon \lambda}$ в (28) теряет энергию на излучение гравитонов, то поля $\Phi^{\varepsilon \lambda}$ в этом уравнении будут представлять собой сумму потенциалов излучения $\psi^{\varepsilon \lambda}$ и потенциалов $\chi^{\varepsilon \lambda}$ остальной части поля, не участвующей в формировании потока излучения, но образуюшей некий фон с “зыбью”. Во втором порядке по полю $\Phi^{\varepsilon \lambda}$, которым здесь вполне можно ограничиться, $\sqrt{-g} t^{\varepsilon \lambda}$ в (28) трансформируется $\mathrm{\kappa} \sqrt{-\gamma} t_{2}^{\varepsilon \lambda}$, определяемому квадратичной формой в $(12)$, и уравнение для $\chi^{\varepsilon \lambda}$ принимает (см. также $[13,14])$ вид

$$
\tilde{\gamma}^{\alpha \beta} D_{\alpha} D_{\beta} \tilde{\chi}^{\varepsilon \lambda}+\mu^{2} \sqrt{-\gamma} \tilde{\chi}^{\varepsilon \lambda}=16 \pi\left[\sqrt{-g} T^{\varepsilon \lambda}+\sqrt{-\gamma}\left(\tau_{1}^{\varepsilon \lambda}+\tau_{2}^{\varepsilon \lambda}\right)\right]
$$

здесь

$$
\begin{aligned}
\tau_{1}^{\varepsilon \lambda} \equiv & \frac{\sqrt{-\gamma}}{16 \pi}\left\{-\gamma^{\alpha \beta} D_{\alpha} D_{\beta} \psi^{\varepsilon \lambda}-\mu^{2} \psi^{\varepsilon \lambda}+\frac{1}{2}\left(\gamma^{\varepsilon \alpha} \gamma^{\lambda \beta}-\frac{1}{2} \gamma^{\varepsilon \lambda} \gamma^{\alpha \beta}\right) \times\right. \\
& \times\left(D_{\alpha} \psi_{\tau}^{\nu} D_{\beta} \psi_{\nu}^{\tau}-\frac{1}{2} D_{\alpha} \psi D_{\beta} \psi\right)-\mu^{2}\left[\psi_{\alpha}^{\varepsilon} \psi^{\lambda \alpha}-\frac{1}{4} \gamma^{\varepsilon \lambda}\left(\psi_{\beta}^{\alpha} \psi_{\alpha}^{\beta}-\frac{1}{2} \psi \psi\right)\right]- \\
& -\gamma^{\varepsilon \beta} D_{\alpha} \psi^{\lambda \nu} D_{\beta} \psi_{\nu}^{\alpha}-\gamma^{\lambda \alpha} D_{\alpha} \psi^{\beta \nu} D_{\beta} \psi_{\nu}^{\varepsilon}+\gamma^{\alpha \beta} D_{\alpha} \psi_{\nu}^{\varepsilon} D_{\beta} \psi^{\lambda \nu}+ \\
& \left.+\frac{1}{2} \gamma^{\varepsilon \lambda} D_{\alpha} \psi_{\nu}^{\beta} D_{\beta} \psi^{\alpha \nu}+D_{\alpha} \psi^{\varepsilon \beta} D_{\beta} \psi^{\lambda \alpha}-\psi^{\alpha \beta} D_{\alpha} D_{\beta} \psi^{\varepsilon \lambda}\right\}
\end{aligned}
$$


а все члены $\tau_{2}^{\varepsilon \lambda}$ обязательно содержат $\chi^{\alpha \beta}$.

Чтобы выяснить, как учесть факт распространения гравитонов в пространстве с римановой метрикой, а не в пространстве Минковского (см. раздел 1), рассмотрим сначала асимптотику потенциалов излучения $\psi^{\varepsilon \lambda}$ вдали от источника. При наличии фундаментальной метрики $\gamma_{\alpha \beta}$ все гравитационные поля трактуются как поля, определяемые в пространстве с этой метрикой. Следовательно, асимптотика $\psi^{\varepsilon \lambda}$ должна иметь вид

$$
\psi_{\mathrm{ac}}^{\varepsilon \lambda} \simeq \frac{1}{r} \sum_{\omega} a_{\omega}^{\varepsilon \lambda} \exp \left\{-i \omega\left(t-\frac{r}{v}\right)\right\} \equiv \frac{1}{r} \sum_{\omega} a_{\omega}^{\varepsilon \lambda} \exp \left(-i \gamma_{\alpha \beta} \varkappa^{\alpha} x^{\beta}\right) \equiv \sum_{\omega} \psi_{\omega}^{\varepsilon \lambda},
$$

где $a_{\omega}^{\varepsilon \lambda}-$ амплитуды парциальных волн $\psi_{\omega}^{\varepsilon \lambda}$, а $\varkappa^{\alpha}$ идентифицируются с 4-импульсами гравитонов. Учитывая (31) в линейной по $\psi^{\varepsilon \lambda}$ части (30) и сохраняя при взятии производных $D_{\alpha}$ только основные члены, получим

$$
-\gamma^{\alpha \beta} D_{\alpha} D_{\beta} \psi_{\mathrm{ac}}^{\varepsilon \lambda}-\mu^{2} \psi_{\mathrm{ac}}^{\varepsilon \lambda} \simeq \sum_{\omega}\left(\gamma_{\alpha \beta} \varkappa^{\alpha} \varkappa^{\beta}-\mu^{2}\right) \psi_{\omega}^{\varepsilon \lambda}
$$

Так как импульсы $\varkappa^{\alpha}$ гравитонов должны удовлетворять уравнению

$$
g_{\alpha \beta} \varkappa^{\alpha} \varkappa^{\beta}=\mu^{2}
$$

то (32) будет равным

$$
\begin{aligned}
\sum_{\omega}\left(\gamma_{\alpha \beta} \varkappa^{\alpha} \varkappa^{\beta}-\mu^{2}\right) \psi_{\omega}^{\varepsilon \lambda} & =\sum_{\omega}\left(\gamma_{\alpha \beta}-g_{\alpha \beta}\right) \varkappa^{\alpha} \varkappa^{\beta} \psi_{\omega}^{\varepsilon \lambda} \simeq \\
& \simeq \sum_{\omega}\left(\Phi_{\alpha \beta}-\frac{1}{2} \Phi \gamma_{\alpha \beta}\right) \varkappa^{\alpha} \varkappa^{\beta} \psi_{\omega}^{\varepsilon \lambda} \simeq \\
& \simeq-\frac{1}{2} \mu^{2}\left(\psi_{\mathrm{ac}}+\chi\right) \psi_{\mathrm{ac}}^{\varepsilon \lambda}-\left(\psi_{\mathrm{ac}}^{\alpha \beta}+\chi^{\alpha \beta}\right) D_{\alpha} D_{\beta} \psi_{\mathrm{ac}}^{\varepsilon \lambda} .
\end{aligned}
$$

Все это указывает на то, что уравнение для потенциалов излучения $\psi^{\varepsilon \lambda}$, задаваемых в пространстве Минковского и определяемых нестатической частью $T_{1}^{\varepsilon \lambda}$ источника $T^{\varepsilon \lambda}$, должно быть нелинейным.м и взятым в виде

$$
\sqrt{-\gamma}\left(g_{\alpha \beta} D^{\alpha} D^{\beta} \widetilde{\psi}^{\varepsilon \lambda}+\mu^{2} \widetilde{\psi}^{\varepsilon \lambda}\right)=16 \pi \sqrt{-g} T_{1}^{\varepsilon \lambda},
$$

где индексы у $D^{\alpha}$ или $D_{\alpha}$ опускаются и поднимаются с помощью метрики $\gamma_{\alpha \beta}$. Легко убедиться, что асимптотика (31) удовлетворяет соответствуюшему (34) однородному уравнению, действительно отвечаюшему закону (33) распространения гравитонов в римановом пространстве. Учитывая (34) в (29), придем к уравнению

$$
\tilde{\gamma}^{\alpha \beta} D_{\alpha} D_{\beta} \tilde{\chi}^{\varepsilon \lambda}+\mu^{2} \sqrt{-\gamma} \widetilde{\chi}^{\varepsilon \lambda}=16 \pi\left[\sqrt{-g} T_{0}^{\varepsilon \lambda}+\sqrt{-\gamma}\left(\tau_{\mathrm{rad}}^{\varepsilon \lambda}+\tau_{3}^{\varepsilon \lambda}\right)\right],
$$

в котором $T_{0}^{\varepsilon \lambda}$ дается статической частью $T^{\varepsilon \lambda}, \tau_{3}^{\varepsilon \lambda}$ обязательно содержит потенциалы $\chi^{\alpha \beta}, \mathrm{a}$

$$
\begin{aligned}
\tau_{\text {rad }}^{\varepsilon \lambda} \equiv & \frac{\sqrt{-\gamma}}{16 \pi}\left\{\frac { 1 } { 2 } \left[\left(\gamma^{\varepsilon \alpha} \gamma^{\lambda \beta}-\frac{1}{2} \gamma^{\varepsilon \lambda} \gamma^{\alpha \beta}\right)\left(D_{\alpha} \psi_{\tau}^{\nu} D_{\beta} \psi_{\nu}^{\tau}-\frac{1}{2} D_{\alpha} \psi D_{\beta} \psi\right)+\right.\right. \\
& \left.+\gamma^{\alpha \beta} \psi D_{\alpha} D_{\beta} \psi^{\varepsilon \lambda}\right]-\mu^{2}\left[\psi_{\alpha}^{\varepsilon} \psi^{\lambda \alpha}-\frac{1}{4} \gamma^{\varepsilon \lambda}\left(\psi_{\beta}^{\alpha} \psi_{\alpha}^{\beta}-\frac{1}{2} \psi \psi\right)\right]- \\
& -\gamma^{\varepsilon \beta} D_{\alpha} \psi^{\lambda \nu} D_{\beta} \psi_{\nu}^{\alpha}-\gamma^{\lambda \alpha} D_{\alpha} \psi^{\beta \nu} D_{\beta} \psi_{\nu}^{\varepsilon}+\gamma^{\alpha \beta} D_{\alpha} \psi_{\nu}^{\varepsilon} D_{\beta} \psi^{\lambda \nu}+ \\
& \left.+\frac{1}{2} \gamma^{\varepsilon \lambda} D_{\alpha} \psi_{\nu}^{\beta} D_{\beta} \psi^{\alpha \nu}+D_{\alpha} \psi^{\varepsilon \beta} D_{\beta} \psi^{\lambda \alpha}-2 \psi^{\alpha \beta} D_{\alpha} D_{\beta} \psi^{\varepsilon \lambda}\right\} .
\end{aligned}
$$


Выражение (36) и следует считать плотностью тензора энергии-импульса гравитационного поля излучения с ненулевой массой гравитонов. Как видно, поправка на искривление пространства приводит к тому, что плотность тензора энергии-импульса части поля определяется не плотностью тензора поля в целом, а содержит вклад от линейной (в пространстве Минковского) комбинации этой части, обусловленный искривлением (или самодействием гравитонов).

Покажем, что даваемая (36) плотность потока излучения (а именно она связана с энергетическими потерями источника) является положительно-определенной. Для этого рассмотрим случай, когда потери энергии на излучение малы и источник можно считать квазистационарным. В таком приближении вместо $\tau_{\mathrm{rad}}^{\varepsilon \lambda}$ можно рассматривать его усредненное по времени значение, которое далее и понимается под $\tau_{\mathrm{rad}}^{\varepsilon \lambda}$. Учитывая, что при взятой точности потенциалы $\psi^{\varepsilon \lambda}$ тоже должны удовлетворять полевому условию (10), часть членов в (36) можно свести к 4-дивергенциям. Такие величины не дадут вклада в усредненное $\tau_{\text {rad }}^{\varepsilon \lambda}$, поэтому они могут быть опушены. Чтобы не превысить взятую по полю точность, в квадратичных по $\psi^{\varepsilon \lambda}$ комбинациях должно использоваться линейное $\left(\right.$ с $g_{\alpha \beta} \rightarrow \gamma_{\alpha \beta}$ и $\left.\sqrt{-g} \rightarrow \sqrt{-\gamma}\right)$ приближение уравнения (34). Это позволяет отбросить в $(36)$ вне источника еще часть членов. В итоге вне источника $\tau_{\text {rad }}^{\varepsilon \lambda}$ примет (см. также $[13,14])$ вид

$$
\tau_{\text {rad }}^{\varepsilon \lambda}=\frac{\sqrt{-\gamma}}{32 \pi}\left[\gamma^{\varepsilon \alpha} \gamma^{\lambda \beta}\left(D_{\alpha} \psi_{\tau}^{\nu} D_{\beta} \psi_{\nu}^{\tau}-\frac{1}{2} D_{\alpha} \psi D_{\beta} \psi\right)-\mu^{2} \psi \psi^{\varepsilon \lambda}\right] .
$$

Следовательно, интенсивность излучения массивных гравитонов будет определяться (в галилеевых координатах) выражением

$$
I=\frac{-1}{32 \pi} \oint_{S \rightarrow \infty}\left[\partial_{0} \psi_{\beta}^{\alpha} \partial_{k} \psi_{\alpha}^{\beta}-\frac{1}{2} \partial_{0} \psi \partial_{k} \psi-\mu^{2} \psi \psi_{0 k}\right] d \sigma^{k}
$$

в котором $\psi^{\varepsilon \lambda}$ подчинены уравнению $(34)$ в линейном по полю приближении. При $r \rightarrow \infty$ его запаздываюшим решением будет

$$
\psi^{\varepsilon \lambda} \simeq \frac{4}{r} \sum_{\nu=-\infty}^{\infty} T_{1}^{\varepsilon \lambda}(\varkappa) \exp (-i \omega t+i \varkappa \mathbf{r})
$$

где $\varkappa \equiv \mathbf{n} \eta \omega, \eta \equiv\left[1-(\mu / \omega)^{2}\right]^{1 / 2}, \mathbf{n} \equiv \mathbf{r} / r, \omega \equiv \nu \omega_{0}, \omega_{0} \equiv \omega_{\min }>\mu, \mathbf{a}$

$$
T_{1}^{\varepsilon \lambda}(\varkappa) \equiv \int \frac{d\left(\omega_{0} t\right)}{2 \pi} d^{3} x T_{1}^{\varepsilon \lambda}(\mathbf{r}, t) \exp (i \omega t-i \varkappa \mathbf{r})
$$

Заметим попутно, что в случае свободного (без источников) гравитационного поля выражение (37) будет характеризовать плотность тензора энергии-импульса бегущих волн, наряду с которыми обязательно будет существовать и фон $\chi^{\varepsilon \lambda}$, определяемый уравнением (29) с $T^{\varepsilon \lambda} \equiv 0$. Учитывая (39) в (37), получим (ср. с (18))

$$
\frac{d I}{d \Omega}=\frac{1}{16 \pi} \sum_{\nu=1}^{\infty} \omega^{2} \eta\left\{\stackrel{*}{a}_{\lambda}^{\varepsilon}(\varkappa) a_{\varepsilon}^{\lambda}(\varkappa)-\frac{1}{2} \stackrel{*}{a}(\varkappa) a(\varkappa)-\frac{\mu^{2}}{2 \eta \omega^{2}}\left[\stackrel{*}{a}(\varkappa) a_{0}^{3}(\varkappa)+\stackrel{*}{a}_{0}^{3}(\varkappa) a(\varkappa)\right]\right\},
$$


где согласно $(39) a^{\varepsilon \lambda}(\varkappa)=4 T_{1}^{\varepsilon \lambda}(\varkappa)$, а индексу 3 , как и в разделе 1 , сопоставляется направление импульса $\varkappa$. Пользуясь далее связями $(11)$, приведем $(40)$ к виду $[13,14]$

$$
\begin{aligned}
\frac{d I}{d \Omega} & =\frac{1}{8 \pi} \sum_{\nu=1}^{\infty} \omega^{2} \eta\left\{\left|a_{2}^{1}\right|^{2}+\frac{1}{4}\left|a_{1}^{1}-a_{2}^{2}\right|^{2}+\frac{\mu^{2}}{\omega^{2}}\left(\left|a_{3}^{1}\right|^{2}+\left|a_{3}^{2}\right|^{2}\right)+\frac{3 \mu^{4}}{4 \omega^{4}}\left|a_{3}^{3}\right|^{2}\right\}= \\
& =\frac{2}{\pi} \sum_{\nu=1}^{\infty} \omega^{2} \eta\left\{\left|T_{1}^{12}\right|^{2}+\frac{1}{4}\left|T_{1}^{11}-T_{1}^{22}\right|^{2}+\frac{\mu^{2}}{\omega^{2}}\left(\left|T_{1}^{13}\right|^{2}+\left|T_{1}^{23}\right|^{2}\right)+\frac{3 \mu^{4}}{4 \omega^{4}}\left|T_{1}^{33}\right|^{2}\right\} .
\end{aligned}
$$

В отличие от (20) это выражение является строго положительно-определенным, а из изложенного следует, что таковым его сделал учет самодействия гравитонов, связанного, в свою очередь, с наличием в составе поля скалярной компоненты, обеспечиваюшей неравенство $\psi$ нулю.

Обратим внимание, что хотя скалярная примесь, связанная со следом $\psi$ (значит, и $a$ ), участвует в формировании потока, сам след $a$, т.е. и след фурье-образов нестатической части плотности тензора энергии-импульса вещества $T_{1} \equiv T_{1}^{\alpha \beta} \gamma_{\alpha \beta}$, в полный поток (41) не входит явно. Однако, если его разбить по независимым спиновым состояниям, то парциальный вклад скалярной примеси выделится ${ }^{6}$. Действительно, учитывая в (41) разбиения (21), получим

$$
\begin{aligned}
\frac{d I}{d \Omega}= & \frac{1}{8 \pi} \sum_{\nu=1}^{\infty} \omega^{2} \eta\left\{\left|b_{2}^{1}\right|^{2}+\frac{1}{4}\left|b_{1}^{1}-b_{2}^{2}\right|^{2}+\frac{\mu^{2}}{\omega^{2}}\left(\left|b_{3}^{1}\right|^{2}+\left|b_{3}^{2}\right|^{2}\right)+\right. \\
& \left.+\frac{3 \mu^{4}}{4 \omega^{4}}\left|b_{3}^{3}\right|^{2}+\frac{1}{12}|c|^{2}+\frac{\mu^{2}}{4 \omega^{2}}\left(b_{3}^{3} c+b_{3}^{3} c\right)\right\}
\end{aligned}
$$

Отсюда видно, что пропорциональный $\left|a_{3}^{3}\right|^{2}$ член в (41) обеспечивается суммарным вкладом спиновых состояний $s_{3}=0$ и $s=0$. Если разность фаз фурье-амплитуд потенциалов излучения, соответствуюших $s_{3}=0$ и $s=0$, хаотична, то последний член в (42) можно опустить.

Поскольку при выводе (36) область определения потенциалов излучения $\psi^{\varepsilon \lambda}$ не ограничивалась внешней по отношению к источнику областью, то этим можно воспользоваться, чтобы интенсивность излучения связать с работой источников. Для этого вычислим 4-дивергенцию от $\tau_{\text {rad }}^{0 \lambda}$ и учтем в полученном результате уравнение $(34)$ в линейном приближении. В итоге найдем

$$
\begin{aligned}
\partial_{\lambda} \tau_{\text {rad }}^{0 \lambda}= & \frac{\sqrt{-\gamma}}{2}\left\{T_{1}^{\alpha \beta} \partial_{0} \psi_{\alpha \beta}-\frac{1}{2} T_{1} \partial_{0} \psi+\frac{1}{16 \pi} \partial_{\lambda}\left(\psi \square \psi^{0 \lambda}\right)-\right. \\
& \left.-2 T_{1}^{\alpha \beta} \partial_{\alpha} \psi_{\beta}^{0}-\partial_{\lambda} \partial_{\alpha} \partial_{\beta}\left(\psi^{\alpha \beta} \psi^{0 \lambda}\right)\right\} .
\end{aligned}
$$

Интегрируя его по объему, получим уравнение баланса

$$
\begin{aligned}
\frac{\partial W}{\partial t}+I= & \frac{1}{2} \int_{V} d^{3} x\left[T_{1}^{\alpha \beta} \partial_{0} \psi_{\alpha \beta}-\frac{1}{2} T_{1} \partial_{0} \psi+\frac{1}{16 \pi} \partial_{\lambda}\left(\psi \square \psi^{0 \lambda}\right)-\right. \\
& \left.-2 T_{1}^{\alpha \beta} \partial_{\alpha} \psi_{\beta}^{0}-\partial_{\lambda} \partial_{\alpha} \partial_{\beta}\left(\psi^{\alpha \beta} \psi^{0 \lambda}\right)\right],
\end{aligned}
$$

\footnotetext{
6) В (41) он скрыт в амплитудах $a_{3}^{3}$, связанных с амплитудами фурье-образов потенциалов, отвечающих состояниям с нулевой проекцией спина два на импульс и нулевым спином.
} 
в котором

$$
W \equiv \int_{V} d^{3} x \tau_{\mathrm{rad}}^{00}, \quad I \equiv \oint_{S} \tau^{0 k} d \sigma^{k} .
$$

В случае квазистационарных источников изменением со временем взятых по объему интегралов можно пренебречь. Усредняя же оставшееся по времени, придем к равенству

$$
I=\frac{1}{2} \int_{V} d^{3} x\left(T_{1}^{\alpha \beta} \partial_{0} \psi_{\alpha \beta}-\frac{1}{2} T_{1} \partial_{0} \psi\right)+\frac{\mu^{2}}{32 \pi} \oint_{S \rightarrow \infty} \psi \psi_{0 k} d \sigma^{k}
$$

Здесь первый интеграл представляет собой работу источников, а второй учитывает результат самодействия гравитонов. Подстановкой в объемный интеграл в качестве $\psi_{\alpha \beta}$ и $\psi$ полуразности запаздывающих и опережающих потенциалов (выделяюшей волновые решения) это выражение для $I$ легко свести к (41).

Наконец отметим, что получаемые в рамках справедливости условий постановки задачи об излучении и взятого приближения метрические коэффициенты $g_{\alpha \beta}$ удовлетворяют сформулированному в [10] принципу причинности. Такое заключение можно сделать уже на основании того, что при вытекающих из условий постановки задачи ограничениях нестатические решения являются поправочными к статическому, а последнее, как известно, принципу причинности удовлетворяет.

\section{4. ЗАКЛЮЧЕНИЕ}

Главный вывод, который можно сделать исходя из изложенного, состоит в том, что энергетические потери источника на излучение массивных гравитонов являются вопреки утверждению авторов [1] положительно-определенными (см. (41), (42)). Это значит, что теория гравитации с ненулевой массой гравитона, если таковой наделила его природа, имеет такие же права на существование, как и теория безмассового поля. Основные уравнения этой теории приведены в разделе 2 (см. (26), (27)).

Получавшиеся ранее отрицательные вклады в интенсивность излучения (см. (20), (22)) являлись следствием отождествления пространства, в котором распространяются рождаемые гравитоны, с пространством Минковского (см. раздел 1). Это заблуждение сохраняется уже более 50 лет. В применявшихся методах упускалось из вида, что в действительности гравитоны распространяются в пространстве с римановой метрикой, индуцируемой полем, в том числе и собственным полем гравитонов. В разделе 3 показано, что учет искривления пространства (или самодействия поля, а, значит, и гравитонов) приводит к положительной определенности энергетических потерь (см. (41), (42)). Как следует из (42), вклад в излучение дают все пять состояний со спином два и состояние с нулевым спином. В случае сферически-симметричного источника излучение массивных гравитонов не исчезает, но испускаться будут только гравитоны в состояниях с нулевой проекцией спина два на импульс и нулевым спином. Установлена связь потока излучения с работой источников (см. (43)).

Отличие массы гравитона от нуля ведет и к другим важным следствиям. Например, наличие массового члена в динамических уравнениях поля приводит (см. [12]) в случае статической сферически-симметричной задачи к несовпадению метрического коэффициента $g_{00}$ (при $\left.d t^{2}\right)$ с обратным значением метрического коэффициента $g_{11}\left(\right.$ при $\left.d r^{2}\right)$ и, как следствие, к невозможности проникновения падающих на центр частиц под сферу Шварцшильда. В силу полноты системы уравнений (27) вместе с уравнением состояния вешества картина эволюции однородной изотропной Вселенной получается (см. [15]) 
в такой теории однозначно определенной. Масса гравитона связывается при этом со значениями параметра замедления и функции Хаббла. Она оценивается величиной порядка $10^{-67}$ г. Наличие массы ведет (см. [15]) к периодическим (с периодом порядка $7,5 \cdot 10^{10}$ лет) пульсациям Вселенной между состояниями с минимальной

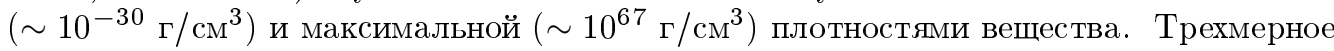
пространство при этом всегда остается евклидовым. Предсказывается скрытая масса, превышаюшая примерно в 25 раз массу видимую. Таким образом, подводя итоги, можно сказать, что реабилитация теории гравитации с ненулевой массой гравитона имеет нетривиальные следствия. Правда, из-за чрезвычайной ее малости во многих случаях она практически не будет сказываться. Например, все результаты [16], связанные с гипотезой о сушествовании гравитационных взаимодействий с нарушенными $C$ и $P$-четностями, останутся в силе, т.к. рассматриваемые там области далеки от тех, где может сказаться массовый член.

Работа выполнена при поддержке Российского фонда фундаментальных исследований.

\section{Список литературы}

[1] Boulware D. G., Deser S. // Phys. Rev. D. 1972. V. 6. №12. P. 3368-3382.

[2] Pauli W., Fierz M. // Proc. Roy. Soc. 1939. V. A73. P. 211; Thirring W.// Ann. Phys. 1961. V. 16. P. 96.

[3] Freund P. G. O., Maheshwari A., Schonberg E. // Astroph. J. 1969. V. 157. P. 857-867.

[4] Fronsdal C. // Suppl. Nuovo Cim. 1958. V. 9. P. 416-443.

[5] Barnes K. J.// J. Math. Phys. 1965. V. 6. P. 788-794.

[6] Ландау Л. Д., Лифииц Е. М. Теория поля. М.: Наука, 1988.

[7] Логунов А.А., Мествиришвили М.А. Релятивистская теория гравитации. М.: Наука, 1989.

[8] Логунов А.А., Лоскутов Ю.М., Мествиришвили М.А. // УФН. 1988. Т. 155. В. 3. C. $369-396$.

[9] Logunov A.A., Loskutov Yu. M., Mestvirishvili M. A. // Progr. Theor. Phys. 1988. V. 80. №6. P. 1005-1023; Intern. J. of Mod. Phys. A. 1988. V. 3. № 9. P. 2067-2099.

[10] Логунов А. А. // ТМФ. 1994. Т. 101. №1. С. 3-27; УФН. Т. 165. В. 2. С. 187-203.

[11] Логунов А. А., Лоскутов Ю. М. // ДАН СССР. 1989. Т. 305. № 4. С. 848-851.

[12] Лоскутов Ю. М. // ТМФ. 1990. Т. 82. № 2. С. 304-312.

[13] Лоскутов Ю. М. // Вестн. МГУ. Сер. физ.-астр. 1991. № 4. С. 49-54.

[14] Loskutov Yu. M. Proc. of the sixth Marcel Grossmann meeting on Gen. Rel. Part B. Kyoto: Japan, 1991. P. 1658-1660.

[15] Лоскутов Ю. М. // ТМФ. 1993. Т. 94. №3. С. 515-528.

[16] Лоскутов Ю. М. // ЖЭТФ. 1995. Т. 107. В. 2. С. 283-298.

Московский государственный университет

\section{Yu. M. Loskutov \\ POSITIVE DEFINITENESS OF THE GRAVITATIONAL RADIATION INTENSITY IN THE THEORY OF GRAVITY WITH NONZERO GRAVITON MASS}

Gravitational radiation flux from arbitrary spatially bounded source is positively defined in the considered theory of gravity with a nonzero graviton mass. A link between energy losses by emission and a work of sources is established. It is shown that the total work contains a part resulted from the interaction of a source with a radiation field and a part resulted from a self-interaction of the field. This is just the sum of two parts that makes the work positively defined in the whole. The general form of a radiation spectrum-angular distribution is obtained taking into account spin and polarization states. For spherically symmetric sources, states with zero spin as well as zero projection on momentum of spin two make contribution into emission. 